\title{
Nuevos conceptos sobre el sistema muscular peribucal
}

\section{News concepts on the peribucal muscular system}

\author{
Giacomotti, J .D.*, Bertone, V.H.**, Conesa, H.A.***, Ouviña, J .M.****, Seiler, J I..*****,

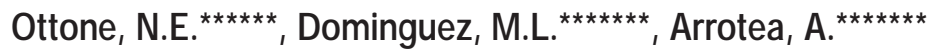

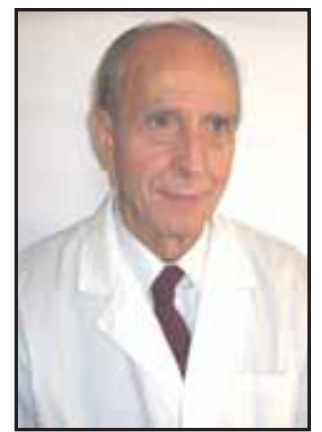

Giacomotti, J .D.

\section{Resumen}

Realizamos una revisión de la musculatura perioral considerándola como un verdadero sistema y analizamos la participación muscular en las distintas funciones de los labios, introduciendo aquí la noción de un músculo buccinador compuesto por dos sectores: uno superior y otro inferior, con acciones e inervación diferentes.

A partir de este enfoque, investigamos la dinámica comisural, a saber: acercamiento (oclusión-proyección labial) y separación de las comisuras, así como también su elevación y descenso. En este aspecto enfatizamos sobre la intervención del sector superior del buccinador (junto a la columna canino-triangular) en la oclusión-proyección labial y la del sector inferior del músculo como integrante del sistema de contención de la saliva al mantener aplicada la mejilla contra la arcada dentaria.

Finalmente remarcamos la presencia de las ramas temporal y cervical del nervio facial señalando los límites de cada una y la importancia que esto representa en el normal funcionamiento del aparato labial.
Abstract

A review of perioral muscles is made considering theme as a real system.

We analyze the muscle involvement in the various functions of the lips, introducing the notion of a buccinator muscle composed by two parts: an upper one and a lower one, with different functions and innervation.

Therefore, the comisural dynamic is investigated, determining the approchement (lip occlusion-projection) and commissure separation as well as its elevation and descend.

It's emphasized that the buccinator upper sector (together with the caninotriangular column) takes part in the lip occlusion -projection and that the buccinator lower sector is a salival containment system which applies the cheek against the dentary arcade.

Finally, it's been highlighted the distribution of the facial nerve branches (cervical and temporal ones) in order to denote their importance in the normal function of the lip complex.

\author{
Palabras clave Musculatura perioral, Músculo buccina- \\ dor, Nervio facial \\ Código numérico 10-104-20
}

$\begin{array}{ll}\text { Key words } & \begin{array}{l}\text { Perioral muscles, Buccinator muscle, } \\ \text { Facial nerve }\end{array} \\ \text { Numeral Gode } & 10-104-20\end{array}$




\section{Introducción}

El preciso conocimiento de la Anatomía es uno de los pilares de la Cirugía Plástica.

Como aporte a tal afirmación realizamos una revisión de los músculos de la pared bucal en armonía con otros autores que enfatizan sobre la existencia de un verdadero sistema periorificial.

El objetivo de este trabajo es realizar un análisis de la arquitectura de la región como paso previo para interpretar correctamente su funcionalidad.

Acto seguido, estudiaremos el sistema involucrado en:

1. Oclusión-proyección labial (acercamiento de comisuras)

2. Ampliación de la hendidura bucal (separación de comisuras)

3. Elevación y descenso de labios y comisuras

4. Inervación pertinente.

Material y método

El material cadavérico utilizado consistió en 30 piezas formolizadas al $40 \%$, provenientes de cadáveres adultos, de raza blanca, abarcando la región mentoniana y obtenidas en bloque con el periostio incluido.

En aquellas piezas que lo permitieron (1) se incluyó el músculo platisma adosado a la piel cervical.

En cada una, y de modo rutinario, se inició la disección desde el plano del periostio hasta la cara profunda de la capa muscular superficial del sistema peribucal, individualizando cada uno de los componentes anatómicos en forma progresiva. Acto seguido, se realizó el abordaje desde la superficie disecando el plano cutáneo y el celular subcutáneo, hasta llegar a la exposición de la cara superficial del conjunto muscular; pasamos entonces a identificar cada uno de los elementos anatómicos integrantes del sistema con ayuda de una lente de aumento de $4 \mathrm{x}$ con leds de iluminación marginal, similar a las utilizadas en joyería o manualidades.

El siguiente paso consistió en la investigación detallada de ambas comisuras, abordándolas primero por la cara posterior del músculo buccinador, pasando luego a explorarlas desde la cara superficial.

Para la disección rutinaria se utilizaron mangos de bisturí de hoja intercambiable $\mathrm{N}^{\circ} 11$ y 15 ; pinzas delicadas tipo Adson, erinas tipo Gilies y tijera Iris de $11 \mathrm{~cm}$.

Para la disección delicada de las comisuras se fabricaron especialmente dos vástagos de madera de $15 \mathrm{~cm}$. de largo por $0,5 \mathrm{~cm}$. de diámetro, cargados en un extremo con sendas agujas BD $13 \times 330 \mathrm{G} \frac{1}{2}$, una de ellas para actuar como elemento de corte mediante el filo del bisel y la otra ligeramente curvada para utilizarla como erina y se utilizó un colposcopio marca Leisegang Berlin West Type bw $772550-60 \mathrm{~Hz}{ }^{\circledR}$ para magnificación.
Accesoriamente se colorearon las ramas del nervio facial (Nervus facialis) con tinta china color sepia, para mejorar la visualización fotográfica.

El material empleado para el registro fotográfico fue:

- Cámara Nikon ${ }^{\circledR}$ Fm - Reflex.

- Película Fuji ${ }^{\circledR}$ para diapositivas color de 100 y 200 asas.

- Objetivo: Nikkon ${ }^{\circledR} 50 \mathrm{~mm} \mathrm{1:14}$

- Lentillas de aproximación $\mathrm{N}^{\circ}$ 1, 2, 3 en todos los casos.

- Digitalización del registro fotográfico anterior (cámara Nikon ${ }^{\circledR}$ ) con Scanner "Genius" Color Page-Vivid ${ }^{\circledR}$ 1200XE - 600dpi.

- Cámara Canon PowerShot A510 ® 3.2Megapixels - Canon Zoom Lens 4x.

Resultados

Coincidimos con la descripción clásica de los músculos elevador del labio superior (M. levator labii superioris) y borla de la barba (M. mentalis), resaltando además, la presencia ocasional de fibras con trayectoria aberrante y escasa significación que pueden aparecer en cualquier punto del sistema.

\section{COLUMNA O COMPLEJO CANINO-TRIAN-} GULAR (Fig. 1, 2, 3, 5).

Estos músculos dan lugar, bilateralmente, a una figura con forma de cinta aplanada dispuesta casi verticalmente entre los maxilares, cercana a la comisura, cubierta por el tejido celular subcutáneo y apoyada sobre el músculo buccinador (M. buccinator).

Este complejo presenta extremos, capas y bordes (2).

Extremo Superior: corresponde a la inserción del canino (M. levator anguli oris) en la fosita homónima de la maxila.

Extremo Inferior: coincide con la base del músculo triangular ( $M$. depressor anguli oris), presentando una línea de inserción oblicua sobre el hueso mandibular. En ocasiones una banda muscular submentoniana une a un extremo inferior con su par contralateral.

Capa Superficial: estas fibras del músculo triangular (M. depressor anguli oris) se inician en el extremo inferior de la columna. Desde aquí siguen un trayecto curvilíneo hasta alcanzar la comisura, desde donde prosiguen como fibras arciformes cortas y largas. Las primeras ascienden oblicuamente por el labio superior hasta el sector nasolabial incisivo del maxilar. Las segundas, en mayor cantidad, ingresan en el labio a cierta distancia de su borde libre. Continúan sin interrupción hacia el lado opuesto alcanzando la columna contralateral, a la que se incorporan para descender describiendo, en totalidad, una curva de concavidad inferior. 


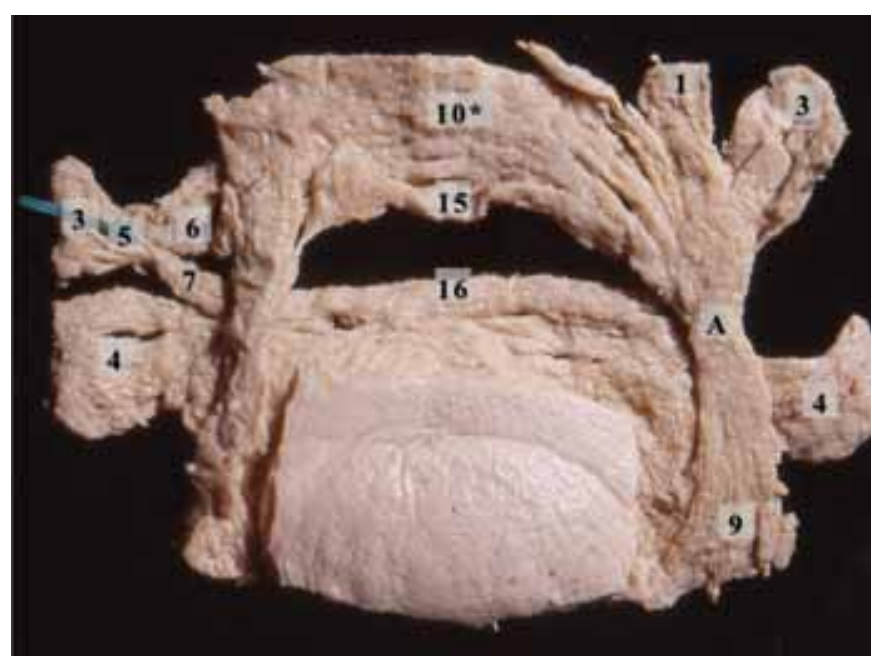

Fig. 1. Pieza extraída en bloque de la región labio-mentoniana conservando parte de la piel en el mentón. Se expone la cara anterior de los planos del sistema muscular peribucal. En el lado derecho se ha evertido la columna canino-triangular para mostrar la "horqueta" del buccinador superior. A. Columna canino-triangular 1. Canino, 3. Buccinador (mitad superior), 4. Buccinador (mitad inferior), 5. Fascículo medio (horqueta) de buccinador superior con: (6) su fascículo labia superior y (7) su fascículo labial inferior. 9. Triangular, 10*. Fibras largas o arciformes del complejo canino-triangular, 15. Borde libre labio superior o bermellón, 16. Borde libre labio inferior o bermellón.

Capa profunda (Fig. 1, 4, 5): constituida por el músculo canino (M levator oris), y desde el extremo superior de la columna, sus fascículos descienden verticalmente apoyados sobre el buccinador (M. buccinator) cubiertos, parcialmente, por las fibras arciformes superiores. A nivel comisural se desprenden fibras arciformes que penetran escalonadamente en el labio inferior alejadas del borde libre del mismo. Continúan su trayecto hasta el complejo opuesto, ascendiendo por él hasta la fosa canina correspondiente configurando otra curva, ahora de concavidad superior.

Borde Externo (Fig. 2): lo constituyen dos clases de fibras: las directas de un maxilar a otro y las que, en reducido número, son aportadas por el cigomático mayor (M. zygomaticus major).

Borde Interno: curvilíneo, abierto hacia la comisura, muestra en su parte central la emergencia de los fascículos arciformes arriba señalados.

\section{CIGOMATICO MAYOR (M. zygomaticus major)} (Fig. 1, 3, 4, 6): a partir de su origen en el malar, desciende oblicuo hacia la zona supracomisural donde emite dos, o a veces tres, órdenes de fibras que son: las descendentes anteriormente mencionadas y las que, pasando entre el complejo canino-triangular y el buccinador (M. buccinator), se incorporan a los labios.

Infrecuentemente se detectan fibras atravesando la capa superficial de la columna.

RISORIO (M. risorius): sumamente inconstante, llega en forma horizontal a cada comisura, lugar en que confunde sus fibras con las de la columna.

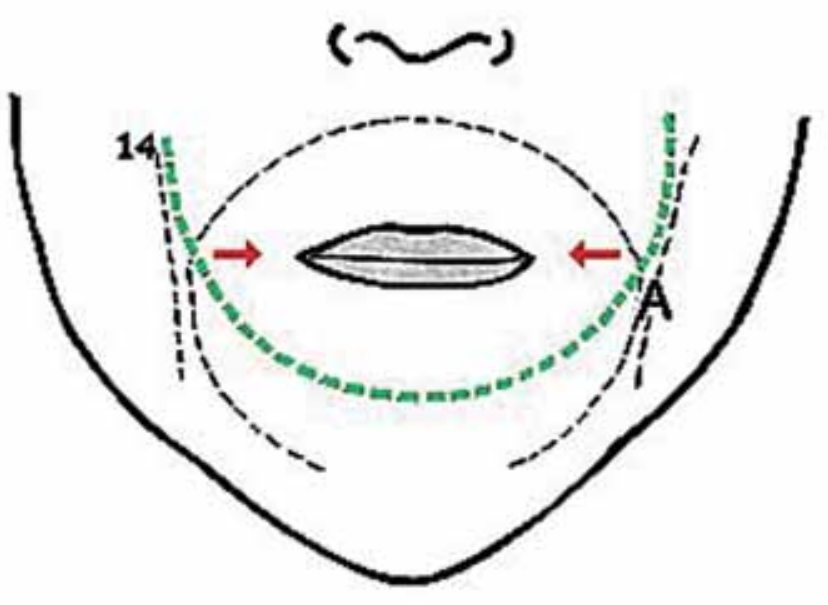

Fig. 2. Esquema del componente anterior del mecanismo de oclusión-proyección (columna canino-triangunlar). A. Columna caninotriangular; 14. Fibras directas del complejo.

PLATISMA (M. platysma) Y CUADRADO DEL MENTON (M. depressor labii inferioris) (Fig. 3, 4, $6,7)$ : el platisma (M. platysma), con origen subcutáneo en el tórax, converge bilateralmente hacia la cara y presenta dos órdenes de fibras: las posteroexternas y las anterointernas.

Las primeras, en menor cantidad, alcanzan al borde externo del complejo caninotriangular donde se entremezclan, pero siempre por debajo del nivel comisural.

Las anterointernas, en mayor número, llegan hasta el hueso mandibular en dos planos: uno superficial y otro profundo. Las superficiales cruzan libremente la maxila, las profundas primero se adhieren al periostio man-

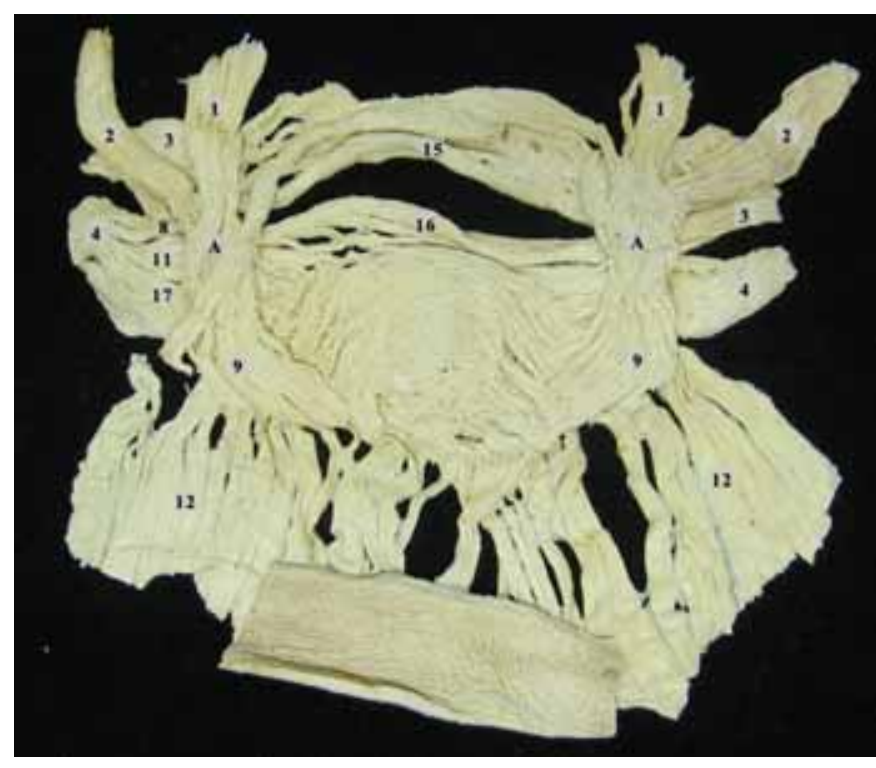

Fig. 3. Pieza extraída en bloque de la región cérvico-mento-labial. En el sector inferior se conserva parte de la piel cervical. Se expone la cara anterior de los planos del sistema muscular peribucal y la del músculo platisma. A. Columna canino-triangular. 1. Canino, 2. Cigomático Mayor, 3. Buccinador (mitad superior), 4. Buccinador (mitad inferior), 8. Fibras superiores, o largas, del buccinador inferior, 9. Triangular de los labios, 11. Fibras medias del buccinador inferior, 12. Platisma, 15. Borde libre labio del superior o bermellón, 16. Borde libre del labio inferior o bermellón, 17. Fibras incisivas del buccinador inferior. 


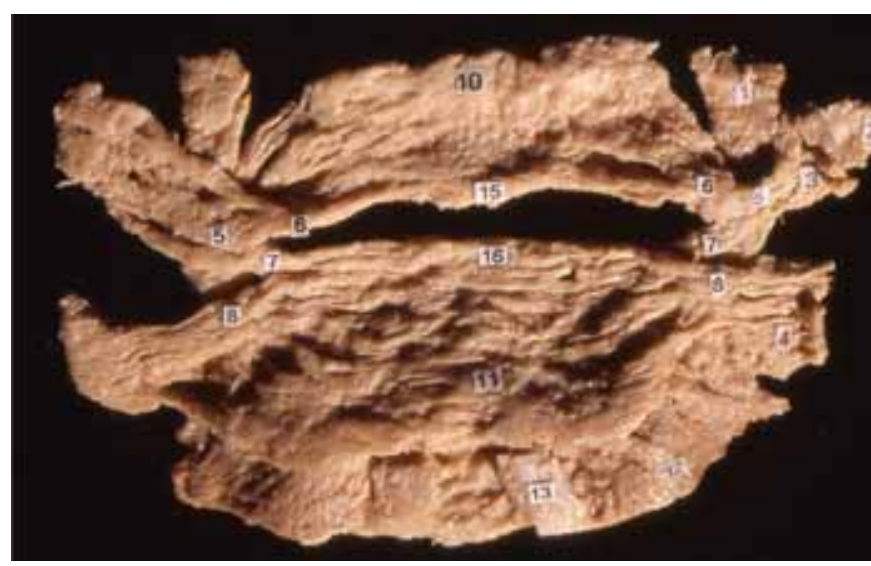

Fig. 4. Pieza que corresponde a la cara posterior de los planos de la figura 1. 1. Canino, 2. Cigomático Mayor, 3. Buccinador (mitad superior), 4. Buccinador (mitad inferior), 5. Fascículo medio (horqueta) de buccinador superior con: (6) su fascículo labial superior y (7) su fascículo labial inferior. 8. Fibras superiores, o largas, del buccinador inferior, 10. Fibras cortas, o incisivas, del buccinador superior, 11. Fibras medias del buccinador inferior, 12. Platisma, 13. Borla del mentón. 15. Borde libre del labio superior o bermellón, 16. Borde libre del labio inferior o bermellón.

dibular, para después continuar junto a las superficiales conformando finalmente el músculo cuadrado del mentón (M. depressor labii inferioris); éste se apoya sobre el buccinador ( $M$. buccinator) y permanece parcialmente cubierto por el complejo caninotriangular.

El destino del cuadrado del mentón (M. depressor labii inferioris) es la piel cercana al borde libre del labio inferior. En el sector mentoniano, se entremezcla con el músculo borla de la barba (M. mentalis).

BUCCINADOR (M. buccinator) (Fig. 1, 4, 5, 6, 7): la serie de disecciones realizadas lo destacan como una franja muscular aplanada compuesta, bilateralmente, por un sector superior y otro inferior.

Mitad o Sector Superior: las fibras nacen en el reborde alveolar de los molares, desde donde se dirigen hacia el plano sagital en forma radiada.

Entre las de posición más elevada, unas buscan el reborde incisivo de la maxila (fibras cortas) y otras, en mayor número y longitud, ingresan en el labio superior recorriéndolo sin interrupción.

Las medias muestran comportamiento clave, pues a distancia variable de la línea media, según el caso, se dividen a modo de "horqueta" en un fascículo superior y otro inferior que, además de formar el ángulo de la boca, pasan a integrar el borde libre o bermellón de ambos labios. Un corte sagital muestra a éstos con disposición en forma de "L" abierta hacia delante (bisel labial). Alcanzado el extremo opuesto, se reagrupan repitiendo en sentido inverso la disposición inicial.

Las inferiores (fascículo diagonal), a veces bien evidentes, cruzan al otro labio.

Mitad o Sector Inferior: sus fibras se desprenden del ligamento ptérigomaxilar para dirigirse horizon-

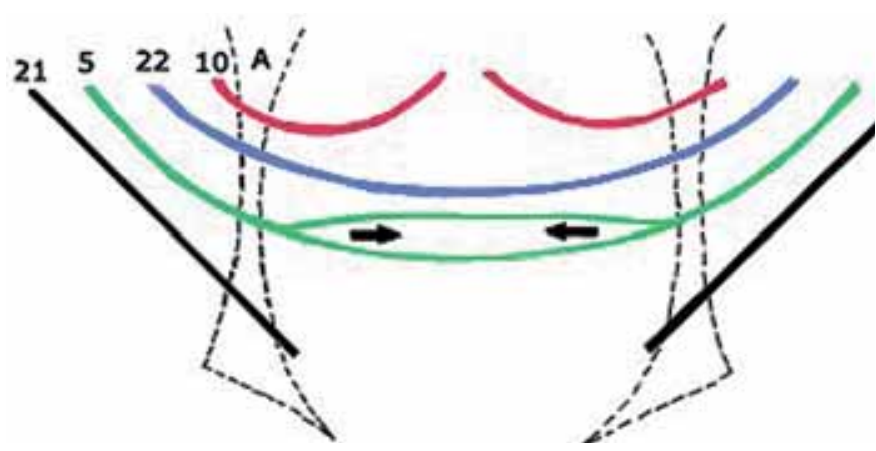

Fig. 5. Esquema del componente posterior del mecanismo de oclusión-proyección (buccinador superior). A. Columna canino-triangular; 5. Fascículo medio (horqueta) de buccinador superior; 10. Fibras cortas, 0 incisivas, del buccinador superior; 21 . Fascículo diagonal de buccinador superior; 22. Fibras largas del buccinador superior.

talmente hacia el plano sagital. Las que se encuentran a nivel comisural pueden separarse también en dos haces (superior e inferior), que se cruzan con los ya descritos, pero con la salvedad de que el superior es inconstante y que, cuando existe, muestra sólo un reducido número de fibras. Las que le siguen, o medias, contribuyen a conformar el cuerpo labial y la mitad inferior de ambas mejillas. Junto a las superiores, terminan en el ligamento contralateral.

Por último, un grupo de fibras más inferiores finaliza después de una corta trayectoria en el reborde alveolar incisivo de la mandíbula.

NERVIO FACIAL (Nervus facialis) (Fig. 6)

$\mathrm{Si}$ bien su distribución resulta plexual se puede considerar que desde la rama témporofacial de cada lado emergen fibras dirigidas a los músculos: cigomático mayor (M. zygomaticus major), elevador del labio superior (M. levator labii superioris), risorio (M. risorius), al complejo canino-triangular y la mitad superior del buccinador (M. buccinator).

La rama cérvicofacial proporciona inervación a los músculos: platisma (M. platysma), cuadrado del mentón (M. depressor labii inferioris), borla de la barba (M. mentalis) y a la mitad inferior del buccinador ( $M$. buccinator). Al investigarla con detenimiento, no vimos esta rama llegando al triangular ni tampoco ir más allá de la zona del buccinador inferior.

\section{Discusión}

El tema de la arquitectura del aparato muscular peribucal ocupa un vasto espectro dentro de la bibliografía consultada y actualmente es considerado como sistema.

Ciertos autores refieren la existencia de dos zonas que son llamadas central y periférica por Rouviere (3), o concéntrica y excéntrica por Casiraghi (4), siendo la periférica/excéntrica (según el autor) la que presenta inserciones óseas. Otros enfocan el problema 


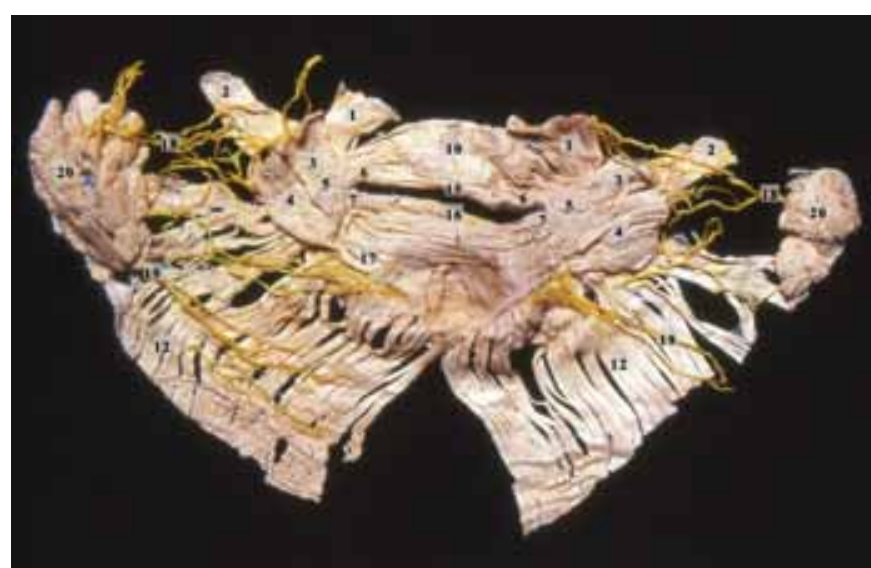

Fig. 6. Pieza extraída en bloque de la región cérvico-mento-labial conservando sectores de ambas glándulas parótidas y exponiendo la cara posterior de los planos del sistema muscular y las ramificaciones del nervio facial. 1. Canino, 2. Cigomático Mayor, 3. Buccinador (mitad superior), 4. Buccinador (mitad inferior), 5. Fascículo medio (horqueta) de buccinador superior con: (6) su fascículo labial superior y (7) su fascículo labial inferior; 10. Fibras cortas, o incisivas, del buccinador superior, 12. Platisma, 15. Borde libre del labio superior o bermellón, 16. Borde libre del labio inferior o bermellón, 17. Fibras incisivas del buccinador inferior, 18. Rama temporofacial del nervio facial, 19. Rama cervicofacial del nervio facial, 20. Parótida.

mencionando mitades superior e inferior (5), o derecha e izquierda (6)

Con respecto al entrecruzamiento fibrilar, existen exposiciones que mencionan, por ejemplo, el producido medialmente en ambos labios $(1,7)$ lo que no es aceptado de forma unánime. También se describe el entrecruzamiento producido en la zona paracomisural entre las fibras del buccinador superior e inferior. El mismo sitio, pero en el plano anterior, es considerado por algunos como lugar de reunión de fibras (7-10); para ellos, este fenómeno da lugar a la formación de un núcleo fibroso o modiolo (modiolus anguli oris) que marcaría el punto de origen o terminación de los músculos canino, triangular y cigomático mayor $(M$. zygomaticus major), explicando su funcionamiento a partir de dicha inserción. Este elemento no pudo ser comprobado en el presente trabajo.

Otra controversia se establece al nombrar la presencia de fibras netamente circulares (5) colocadas muy próximas al orificio bucal, concepto que ha perdido vigencia en la actualidad.

Existen varias teorías sobre la formación del músculo orbicular de la boca. De acuerdo a diversos autores este músculo no es una unidad individual, sino que está formado por la convergencia de los músculos faciales periorales que confluyen en la comisura labial, formando el modiolo (modiolus anguli oris) y desde allí, algunos de ellos, envían fibras a los labios $(8,11)$.

También se describen distintas disposiciones con respecto al músculo buccinador (M. buccinator), a las que se definen como variaciones, y no como su disposición normal. Algunos sostienen que podría disponerse en dos capas y Luschka también menciona que

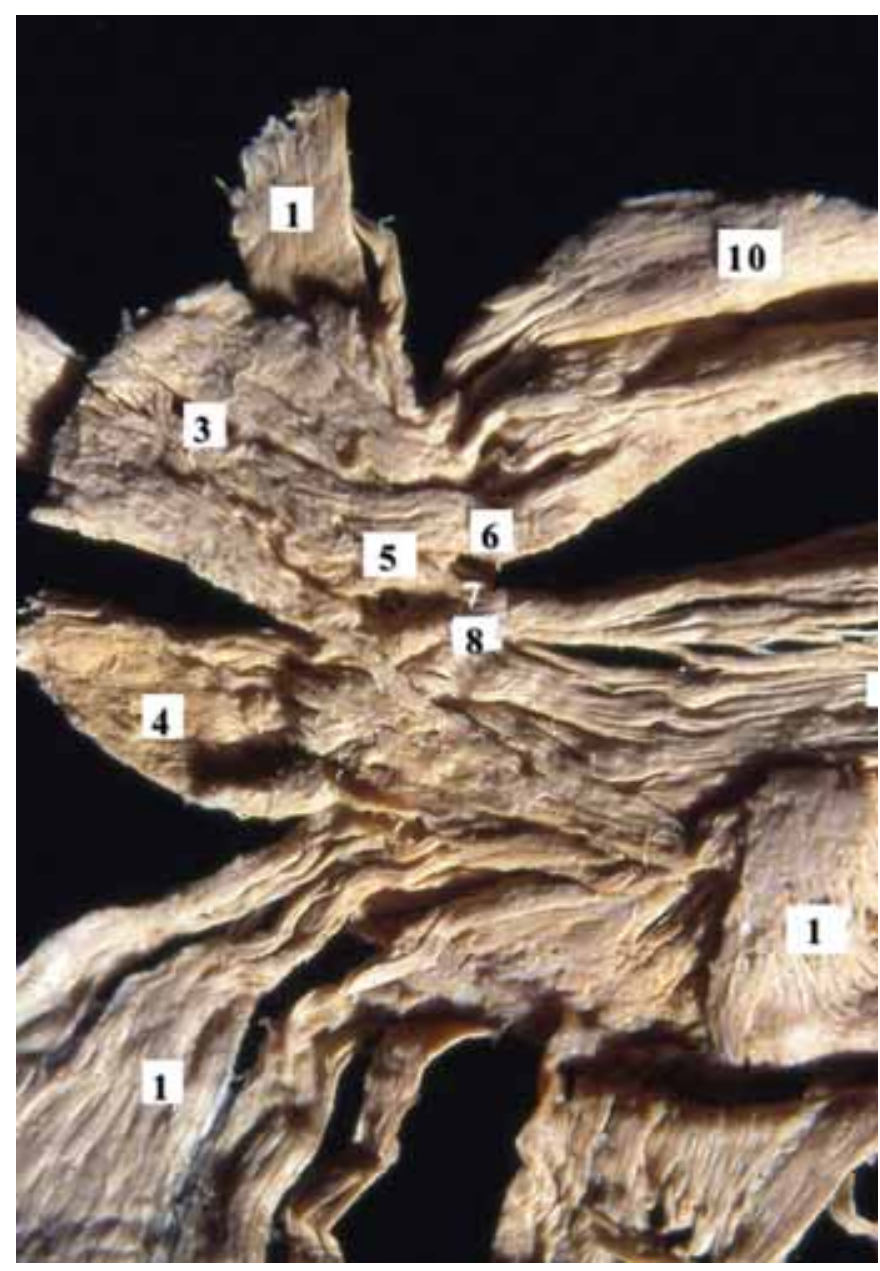

Fig. 7. Pieza que corresponde a la mitad derecha de la Figura 5. Se expone la cara posterior de los planos de esta figural. Canino, 3. Buccinador (mitad superior), 4. Buccinador (mitad inferior), 5. Fascículo medio (horqueta) de buccinador superior con: (6) su fascículo labial superior y (7) su fascículo labial inferior; 8. Fibras superiores, 0 largas, del buccinador inferior, 10. Fibras cortas, 0 incisivas, del buccinador superior, 12. Platisma, 13. Borlas del mentón.

puede continuarse con el constrictor superior de la faringe (M. constrictor pharyngis superior) sin interrupción de un rafe (12). Varias fibras pueden originarse de un tendón separado del músculo temporal (M. temporalis). Por su parte, Cruveihlier y Hyrtl (8) consideran que el buccinador $(M$. buccinator) y el orbicular de la boca (M. orbicularis oris) pueden presentarse como un mismo músculo, por lo que proponen, a esta variación, el nombre de buccolabialis, buccionatolabialis y contrahens buccarum labiorumque. Sin embargo, Cruveilhier sigue afirmando la existencia de un músculo orbicular de la boca independiente al establecer que las fibras del buccinador (M. buccinator) se continúan a lo largo de la parte periférica del orbicular de la boca y se insertan luego del lado opuesto de la mandíbula. También afirma que en el labio inferior, las fibras más inferiores del músculo buccinador (M. buccinator) forman dos tiras, una que se inserta en el lado opuesto de la mandíbula y otras más periféricas, que se insertan justo antes de cruzar la sínfisis. 
D’Andrea y Barbaix (8) por su lado, consideran la existencia de una expansión inferior del músculo buccinador (M. buccinator), una cuarta banda, que pasa profunda al músculo orbicular de la boca y se fusiona con su homóloga contralateral.

Por otro lado, los músculos platisma (M. platysma) y cuadrado del mentón (M. depressor labii inferioris) serían, según Digmon, los verdaderos depresores de labio inferior y de la comisura

En cuanto a la lesión del nervio marginal mandibular (Rama marginalis mandibularis), también llamado nervio de Jaffé por la mayoría de los autores, traería aparejado el descenso de la comisura bucal (13-15).

\section{Conclusiones}

La revisión anatómica de la problemática sobre el dispositivo muscular perioral ha permitido, en una interpretación puramente anatómica, llegar a las siguientes conclusiones:

1. Confirmar la disposición en planos: a) superficial: involucra al canino-triangular; b) intermedio: representado por el cigomático mayor $(M$. zygomaticus major) y el cuadrado del mentón (M. depressor labii inferioris); c) profundo: constituido por las dos mitades del buccinador (M. buccinator).

2. Señalar al complejo canino-triangular y a la mitad superior del músculo buccinador (M. buccinator) como responsables de la oclusión-proyección labial (Fig. 1) mientras que la acción opuesta está a cargo del risorio ( $M$. risorius), del cigomático mayor (M. zygomaticus major) y del buccinador inferior. Este último, además, es el responsable de aplicar la mejilla contra la arcada dentaria inferior.

3. Demostrar que los bordes libres labiales están formados, principalmente, por la división en "horqueta" de las fibras del sector superior del músculo buccinador (M. buccinator).

4. Afirmar que los músculos platisma (M. platys$m a$ ) y cuadrado del mentón ( $M$. depressor labii inferioris) dadas su inervación, dirección y terminación fibrilar son los reales depresores del labio inferior (eversión) y de la comisura.

5. Enfatizar sobre el concepto de la doble inervación que recibe el músculo buccinador (M. buccinator); su mitad superior es inervada por la rama témporofacial mientras que la inferior lo está por el nervio marginal de la rama cérvicofacial (nervio de Jaffé).

El preciso conocimiento de esta disposición resul- ta fundamental para comprender la dinámica comisural ya que una lesión de este nervio determinará, en relación al buccinador inferior, debilitamiento del mecanismo de contención labial (flacidez de la meji1la), lo que da como resultado, la aparición de sialorrea.

En cambio la función de elevación de la comisura permanecerá intacta al no afectarse la inervación del cigomático mayor (M. zygomaticus major) ni la del sistema muscular de la oclusión-proyección (complejo canino-triangular y buccinador superior) por lo cual, el individuo podrá efectuar acciones como, por ejemplo, silbar o besar.

\section{Dirección del autor}

\author{
Dr. José D. Giacomotti \\ Av. Santa Fé 202911 A \\ C1123AAC Ciudad Autónoma de Buenos Aires. \\ Capital Federal. Argentina \\ e-mail: jdgiacomotti@yahoo.com.ar
}

\section{Bibliografía}

1. Romanes G.J. Cunningham: "Tratado de Anatomía", Ed. McGraw-Hill Interamericana, Madrid, 12 a Edición, 1987.

2. Yacomotti J.D. (Giacomotti J.D.), Varela Albieni V., Manrique G., Conesa H.A.: "Previous communications of the sistematization of the oral muscles". XIVth International Symposium on Morphological Sciences, Beijing, China 1997. Bol. Publ. Abs.: 4; P 29, 221.

3. Rouviere H., Delmas A.: "Anatomía Humana. Descriptiva, topográfica y funcional”. Tomo 1: Cabeza y cuello. Editorial Masson. $10^{\circ}$ Edición. Barcelona. 1999.

4. Casiraghi J.C. y col.: "Anatomía del cuerpo humano: funcional y quirúrgica. Tomo 3: Regiones de la cabeza, cuello, tórax y órganos de los sentidos. Editorial Ursino. $1^{\circ}$ Edición. Buenos Aires. 1981.

5. Testut L., Latarjet A.: Anatomía Humana. Tomo $1^{\circ}$. Salvat Editores. Barcelona. 1988.

6. Fernandez Villoria J.M.: "A study of the development of the orbicularis oris muscle". Plast Reconstr Surg. 1975;55(2):205.

7. Williams, W.: "Anatomía de Gray". Editorial Harcourt Brace. $38^{\circ}$ Edición. Madrid. 1998.

8. D'Andrea E., Barbaix E.: "Anatomic research on the perioral muscles, functional matrix of the maxillary and mandibular bones". Surg Radiol Anat. 2006 ;28(3):261.

9. Freilinger G., Gruber H., Happak W., Pechmann U.: "Surgical anatomy of the mimic muscle system and the facial nerve: importance for reconstructive and aesthetic surgery". Plast Reconstr Surg. 1987; 80(5):686.

10. Lightoller G.H.S.: "Facial muscles: the modiolus and muscles surroundin the rima oris with some remarks about the panniculus adiposus" J Anat. 1925; 60(Pt 1): 1.

11. Rubin L.: "The anatomy of a smile: its importance in the treatment of facial paralysis". Plast Reconstr Surg. 1974; 53(4):384.

12. Bergman RA, Afifi AK, Miyauchi R.: "Virtual hospital illustrated encyclopedia of human anatomic variation". Part 1: muscular system. Internet_http://www.anatomyatlases.org/ AnatomicVariants/AnatomyHP.shtml

13. Daane S.P., Owsley J.Q.: "Incidence of cervical branch injury with "marginal mandibular nerve pseudo-paralysis" in patients undergoing face lift". Plast Reconstr Surg. 2003; 111(7): 2414.

14. Ellenbogen R.: "Pseudo-paralysis of the mandibular branch of the facial nerve after platysmal face-lift operation". Plast Reconstr Surg. 1979; 63(3): 364.

15. Savary V., Robert R., Rogez J.M., Armstrong O., Leborgne J.: "The mandibular marginal ramus of the facial nerve: an anatomic and clinical study". Surg Radiol Anat 1997; 19: 69. 